\title{
"A CASE OF TREMA"
}

Authors: María Plaza Yuste, Daniel Fernández Faber, Yanetsy Ainslie Mata, Francisco Javier Torres Varona, Celia Maria Hernández Caro, Jose David Cozar Ortiz, Asuncion Abril Garcia, Jose Luis Perez Iñigo Gancedo, Marta E. Presa Garcia.

Gómez Ulla Military Hospital Glorieta del ejercito sn 28047, Madrid, Spain

\section{Introduction:}

The pre-delusional state is known as a set of psychopathological events that precede delirium and include cognitions, sensations, emotions or actions that can manifest by themselves or in combination.

In order to reduce the duration of untreated psychosis which associates with poor prognosis, interest in early recognition and early intervention in psychosis has grown in the recent years.

\section{Objective:}

To review the evolution of a clinical case from the early onset of symptomatology.

\section{Clinical History:}

The case of a 26-year-old male, who is admitted to Psychiatry Hospitalization because of perception alterations and concentration difficulties.

No medical or past psychiatric history. No actual medical conditions nor actual medical drug treatments.

He was born in Madrid, been the youngest of 2 brothers, he lives with his parents. He has studied a technical architecture degree and is currently studying an opposition to be able to work in the military as an engineer.

$\mathrm{He}$ is referred to psychiatry from Primary Care, because of perception alterations, difficulty concentrating and progressive isolation of several months of evolution.

The patient refers to have abandoned his studies and the academy in which he was preparing the opposition within a few days of starting because of concentrations difficulties and inability to keep up with the rest of his class. Additionally he refers feelings of strangeness, describing situations in his everyday routine that disturb him and that he does not yet understand "... I see the same people more than once when I go out for a walk, like if they repeated themselves... cars accelerate when they pass by me... there are reflections of the sun, it seems that they want to get my attention, but I'm not sure what is happening... things sometimes change color... while I watch TV I see images that I know don't belong to the TV show... I don't know what's going on..."

\section{Psychopathological examination}

Regarding the psychopathological examination the patient was conscious and globally oriented. He had no self-awareness of suffering a mental illness, but was well aware of his own psychological suffering. He

behaved according to usual courtesy standards. Psychomotor retardation was detected. Reduced emotional expression. Maintained attention with concentrations difficulties. Mood characterized by intense anxiety and perplexity. Thought blockages and interceptions, thought content with vague ideas of reference. He describes experiences of

vague significance, and also possible unstructured interpretive phenomena. He denies autolytic ideation. Conciliation insomnia. No anorexia. Limited insight capability. Partially distorted critical judgment.

\section{Complementary tests:}

Blood and urine tests, EEG, Brain CT: no pathological results.

\section{JC: Attenuated psychotic disorder}

\section{Evolution:}

This patient has been monitored for a period of one year and six months on a monthly basis, during which the treatment has been optimized to the minimum effective dose ( Olanzapine $5 \mathrm{mg}$ ) and clinical evolution has

been monitored with the PANS, BPRS and SANS scales. The symptomatology progressively subsided. After a year of treatment, the patient was able to return to the world of work, resume social relations and lead a life like anyone of his age.
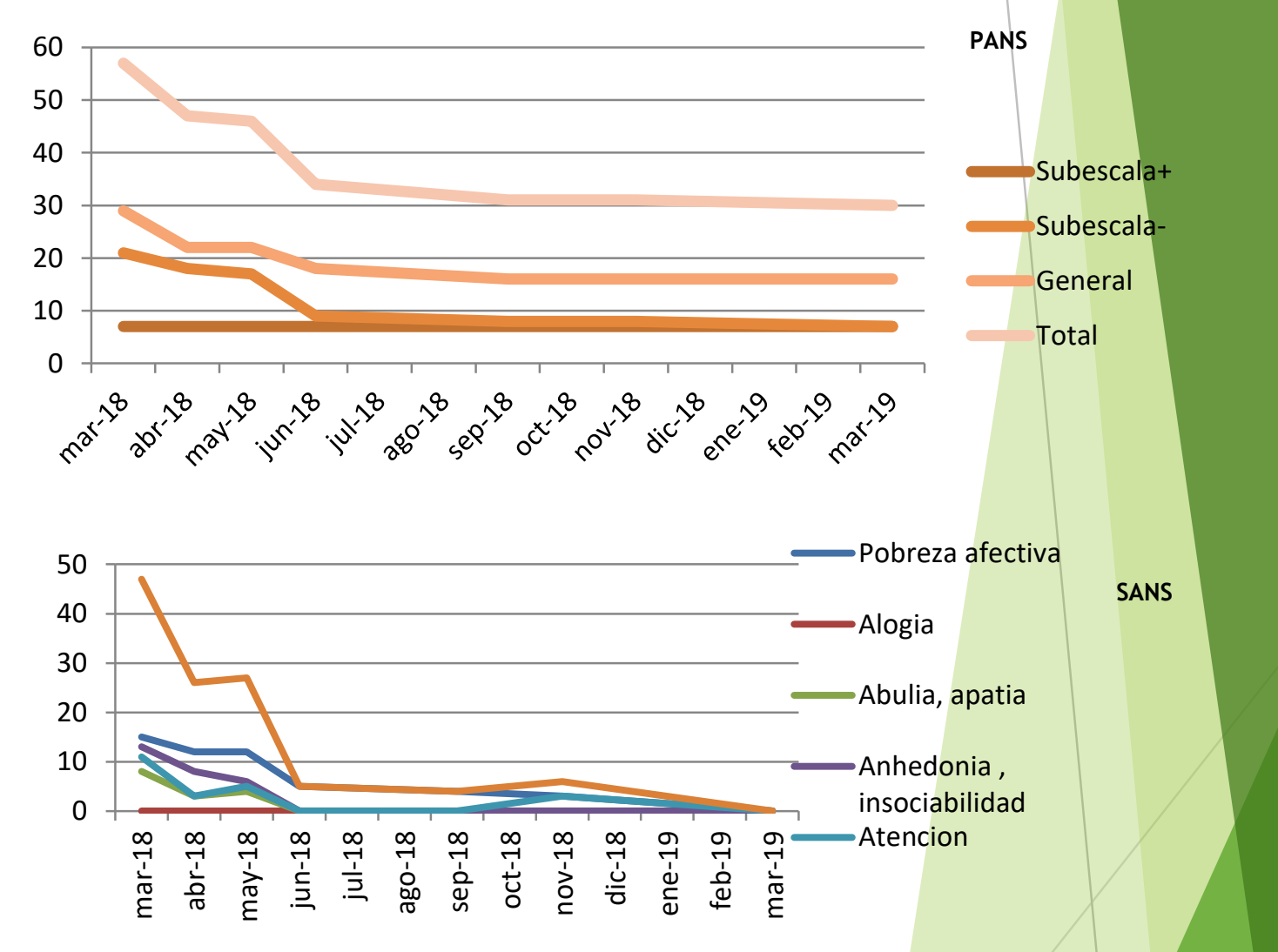

\section{Discussion}

The predelusional state is known as a set of psychopathological events that precede delirium and include alterations in cognitions, sensations,emotions or behavior that can manifest themselves alone or in combination.

In order to reduce the duration of untreated psychosis, interest in early recognition and early intervention in psychosis has grown in the recent years. The prodromal states, also called pre-delusional, have been of wide interest over many years and have been described by the different classical schools of psychiatry; being the most characteristic, the psychiatrist Klaus Conrad with his main work "Die beginnende Schizophrenie. Versuch einer Gestaltanalyse des Wahns" (1958). In this work he develops a Stage Model of Beginning Schizophrenia based on three phases: Trema, apophany and anastrophe.

In the current scientific literature, this prodromal state has adopted the term "mental state of risk". Recent studies have assessed the possibility of detecting these early stage psychotic patients: those with a significant family history of psychosis (Miller et al., 2001), those who have had school and learning difficulties during puberty ( Lin et al.,2017) and those with certain perception alterations ( Klosterkotter et al. 2001).

\section{Conclusions}

Early detection of symptoms that could evolve into severe mental disorder is of great importance.

- Although there is no firm consensus today on how to act on this risk situation "intervention vs no intervention", it is necessary to closely monitor these patients. 